\title{
Where Is the Catheter? Identifying Misplacement of a Dialysis Catheter. Reply to Dr. Zhang et al.
}

\author{
Gioacchino Li Cavoli Onofrio Schillaci Franca Servillo Tancredi Vincenzo Li Cavoli \\ Mattia Palmeri Carmela Zagarrigo \\ Nephrology Dialysis Renal Transplant, ARNAS Civico and Di Cristina Hospital, Palermo, Italy
}

Dear Editor:

We thank Dr. Xiuying Hu and co-authors for their attention to our report on the malposition of a central vein catheter (CVC) for haemodialysis (HD) and we appreciate their considerations on identifying the CVC misplacement in the described case. We accept the remarks regarding the CVC localization in the azygos vein arch with the tip slightly deviated to the left at T5 level. Commonly, a normal azygos vein is so small that it cannot be detected by chest radiography [1]. Furthermore, according to the right practice of CVC positioning, the catheter tip must not lie at a perpendicular angle against the superior vena cava because this generates a risk for vascular erosion [2]. Malposi-

\section{References}

1 Liu Y, Guo D, Li J, Zhang X, He J, Huang M, et al. Radiological features of azygos and hemiazygos continuation of inferior vena cava: A case report. Medicine (Baltimore). 2018 Apr;97(17):e0546. tion occurs in about one-third of CVC insertions if imaging guidance is not used, while it happens less frequently under sonographic or fluoroscopic guidance. The catheter should be placed in a large-calibre vein with a sufficient flow to tolerate infusion, which is usually the inferior or superior vena cava. Misplaced CVC has high probability of clinical and mechanic complications during HD sessions. In our experience, we observed a patient on long-term $\mathrm{HD}$, admitted for pneumonia, after 17 months from the insertion of a CVC for HD. Examining the chest X-ray image, we detected the CVC malposition, confirmed by the CT radiological report. We did not perform further investigations because there were not clinical signs (oedema, deep venous thrombosis, dysrhythmias) or dialysis events (CVC malfunctioning, extracorporeal circuit clotting) suggesting the contrast venography. According to guidelines, chest X-ray is routinely performed to check the correct position or a CVC misplacement. One of the rare courses of the misplaced catheter is the left superior intercostal vein, accessory hemiazygos vein and hemiazygos vein [3]. Furthermore, careful evaluation of the CVC tip must always be carried out to identify a possible malposition. Reporting this case, we wanted to draw the nephrologists care to the correct positioning of the CVC.
2 Zadeh MK, Shirvani A. The role of routine chest radiography for detecting complications after central venous catheter insertion. Saudi J Kidney Dis Transpl. 2014 Sep;25(5): 1011-6.
3 Padovan RS, Paar MH, Aurer I. (Mis)placed central venous catheter in the left superior intercostal vein. Radiol Oncol. 2011 Mar;45(1): 27-30.
Dr. Gioacchino Li Cavoli Nephrology Dialysis Renal Transplant ARNAS Civico and Di Cristina Hospital 43 Via Francesco Cilea, IT-90144 Palermo (Italy) E-Mail gioacchinolicavoli@ libero.it 